\title{
Erratum: Interplay between Kondo effect and Ruderman-Kittel-Kasuya-Yosida interaction
}

Henning Prüser, Piet E. Dargel, Mohammed Bouhassoune, Rainer G. Ulbrich, Thomas Pruschke, Samir Lounis \& Martin Wenderoth

Nature Communications 5:5417 doi: 10.1038/ncomms6417 (2014); Published 11 Nov 2014; Updated 13 Feb 2015

This Article contains typographical errors in equation (1), in which a number of mathematical operators were inadvertently removed during the production process. The correct version of equation (1) appears below.

$$
\widehat{H}=\sum_{\mathbf{k} \sigma} \epsilon_{\mathbf{k}} \hat{n}_{\mathbf{k} \sigma}+\sum_{i \sigma}\left(\epsilon_{d}+\frac{U}{2} \hat{n}_{i,-\sigma}\right) \hat{n}_{i \sigma}+\frac{V}{\sqrt{N}} \sum_{i \mathbf{k} \sigma}\left(e^{i \mathbf{k} \cdot \mathbf{R}_{i}} \hat{c}_{\mathbf{k} \sigma}^{\dagger} \hat{d}_{i \sigma}+h . c .\right)
$$

